\title{
A case of unilateral linear lichen planus: related to orthopedic prosthesis or not?
}

\author{
Müzeyyen Gönül ${ }^{1}$, Seda Atay ${ }^{1}$, Bengu Cevirgen Cemil ${ }^{1}$, Havva Kaya Akış ${ }^{1}$, Aysun Gökçe \\ ${ }^{1}$ Department of Dermatology, Diskapi Yildirim Beyazit Education and Research Hospital, Ankara, Turkey \\ Head of the Department: Müzeyyen Gönül \\ 2Department of Pathology, Dışkapı Yıldırım Beyazıt Education and Research Hospital, Ankara, Turkey \\ Head of the Department: Murat Alper
}

Postep Derm Alergol 2015; XXXII (4): 310-311

DOI: $10.5114 /$ pdia.2015.48046

Lichen planus (LP) is a skin disease characterized by violaceous, polygonal, flat-topped papules and plaques. The form with lichenoid papules in linear distribution is called linear lichen planus (LLP). It is usually distributed unilaterally and follows Blaschko lines. Lichen planus is seen in less than $0.2 \%$ of all LP patients [1]

To our knowledge, there has been no LPP case due to orthopedic implant reported in the literature before. We present a LLP patient because LLP is rarely seen and it might be precipitated by orthopedic endoprosthesis.

A 64-year-old woman presented with a pruritic rash on her right leg. The patient had diabetes mellitus and bilateral blindness due to the diabetic retinopathy. She had undergone an operation because of a femoral bone fracture 1 year ago and an implant including chromium and cobalt was placed in this operation. The lesions appeared over the operation area 7 months after the operation. She was treated with topical steroids without any response. On physical examination she had violaceous and brownish papules that tend to form plaques in a linear distribution from the right hip to the knee (Figure 1). There were no mucosal involvement or nail changes. The histopathological examination revealed wedge-shaped hypergranulosis, destruction of the basal layer, band-like lymphocytic infiltration on dermoepidermal junction, melanin incontinence and melanophages on the upperdermis (Figure 2). A diagnosis of LLP was made according to clinical and histopathological findings. We planned to perform a patch test but the patient refused. Treatment was initiated with a potent topical steroid. The patient did not come for follow-up.

Linear lichen planus is a rare form of LP and represents less than $0.2 \%$ of all LP patients. Linear lichen planus is different from linear configuration which is shorter and thinner of the LP due to the Koebner phenomenon.
The differential diagnosis of LLP includes other dermatoses following the Blaschko lines such as the inflammatory linear verrucous epidermal nevus, lichen striatus, linear lichen nitidus, linear lichenoid drug eruption, linear psoriasis, linear Darier's disease and linear graft-versushost disease [1]. Histopathological examination provided definite diagnosis.

The Blaschko lines are believed to trace the migration of embryonic cells. Some lesions of congenital and acquired skin diseases may follow the Blaschko lines. It is suggested that the acquired dermatoses following the Blaschko lines may occur because of viral infections or medications [2]. In our case we believe that the metalcontaining orthopedic endoprosthesis may be the precipitating factor.

Metal allergy (chromium, cobalt, nickel dependent) is common in the society. It develops because of contact of skin with jewelry, accessories, mobile phones, and similar products. The skin lesions are characterized with erythema, papules, and vesicles that occur after long-term repeated skin contact [3].

There are many oral LP cases reported in the literature due to the amalgam used in dental implants [4]. In a patient with a teeth implant, it was reported that LLP developed on the mandible and the lesions regressed significantly after the removal of this implant [5]. Additionally, 3 patients with dental implant-related LLP were reported. These patients had also positive reactions to gold and mercury on the patch test. In one of these patients, the lesions gradually disappeared within 6 months after removal of dental materials, while in the other 2 cases, oral lesions were treated with disodium cromoglycate even when the dental material was not changed [6].

Orthopedic prosthesis implantation has become more common because of the increase in the aged population. A typical orthopedic implant includes a high amount of

Address for correspondence: Seda Atay, Department of Dermatology, Diskapi Yildirim Beyazit Education and Research Hospital, 06110 Ankara, Turkey, phone: +90 03125963028, e-mail: drsedasayar@gmail.com

Received: 1.08.2014, accepted: 10.09.2014. 


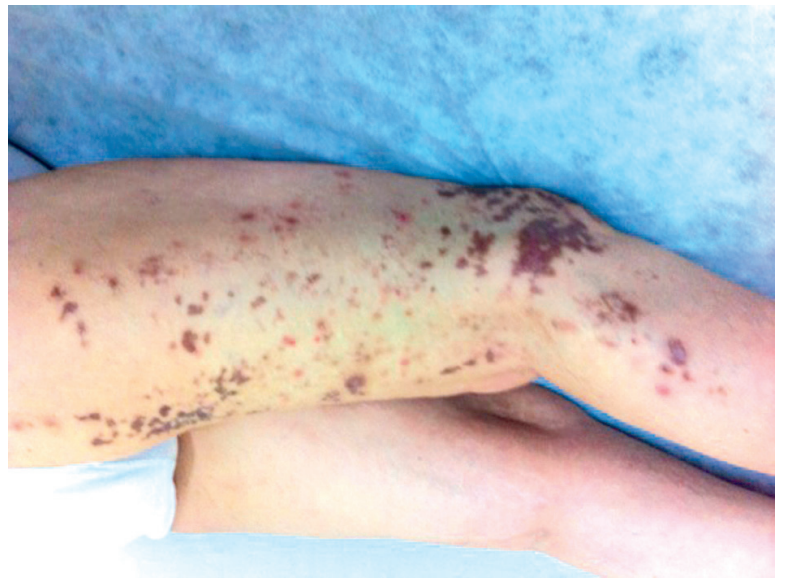

Figure 1. Violaceous and brownish papules tend to form plaques in a linear distribution from the right hip to the knee

chromium, cobalt, and molybdenum and there are many different types. It has been shown that the concentrations of chromium and cobalt were increased in serum and urine tests of patients with implants. Although the incidence of metal allergy is high in these patients, the long-term biological effects of a high metal concentration is still unknown [3]. It is suggested that the metals joining the circulatory system activates the immune system by cytokine release and thus lead to lesion development [5]. The internal orthopedic implant-dependent cutaneous lesions have been known for a long time. These lesions are mostly in eczematous and allergic nature, and rarely as urticarial and bullous vasculitic skin reactions. Also, some complex immune reactions that cause pain at the implant region and even relaxation of the implant can be seen. The metal implants containing nickel, chromium, and cobalt are the most common factors leading to delayed type hypersensitivity reactions [7]. In our case the implant included chromium and cobalt. Although we could not perform a patch test to prove this, we believe that the orthopedic implant in our case caused cytokine release and led to LP lesions as seen with dental materials.

The exact etiology of LP is still unknown but longterm triggers like dental implants have been suggested to cause LP lesions. The appearance time of lesions after implantation and the localization of lesions on the same side as the implant make us believe that chromium or cobalt within the implant may be the trigger factors of LLP lesions in our case.

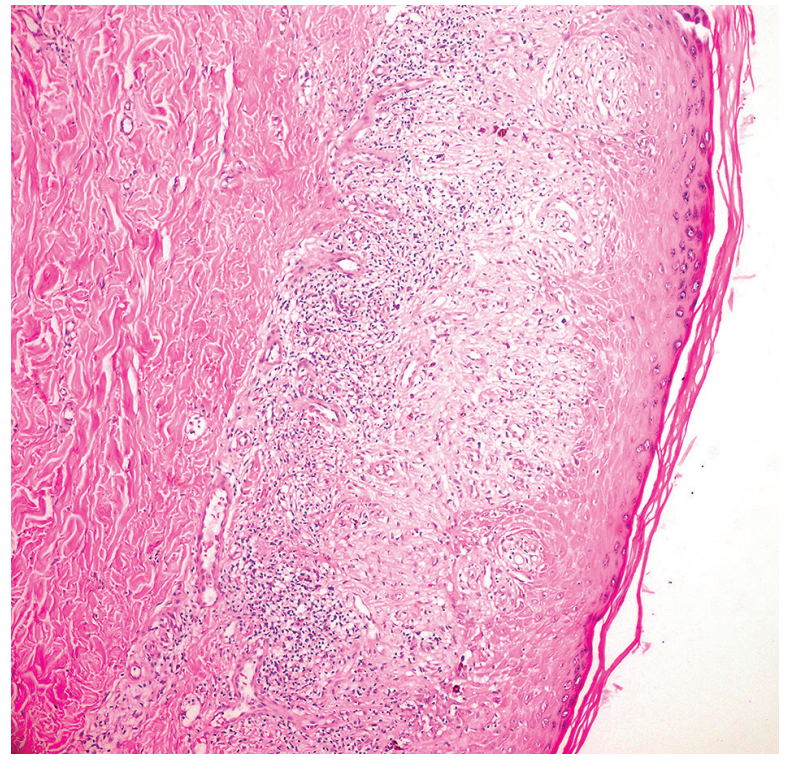

Figure 2. The histopathological examination revealed wedge-shaped hypergranulosis, destruction of the basal layer, band-like lymphocytic infiltration on dermoepidermal junction $(H+E, 100 x)$

\section{Conflict of interest}

The authors declare no conflict of interest.

\section{References}

1. Kabbash C, Laude TA, Weinberg JM, Silverberg NB. Lichen planus in the lines of Blaschko. Pediatr Dermatol 2002; 19: 541-45.

2. Serarslan G, Hakverdi S. Two cases of the linear lichen planus in the lines of blaschko. Turkderm 2008; 42: 28-30

3. Thyssen JP, Jakobsen SS, Engkilde K, et al. The association between metal allergy, total hip arthroplasty, and revision. Acta Orthopaedica 2009; 80: 646-52.

4. McParland H, Warnakulasuriya S. Oral lichenoid contact lesions to mercury and dental amalgam - a review. J Biomed Biotechnol 2012; 2012: 589569.

5. Mizoguchi S, Setoyama M, Kanzaki T. Linear lichen planus in the region of the mandibular nerve caused by an allergy to palladium in dental metals. Dermatology 1998; 196: 268-70.

6. Sasaki G, Yokozeki H, Katayama I, Nisioka K. Three cases of linear lichen planus caused by dental metal compounds. J Dermatol 1996; 23: 890-2.

7. Basko-Plluska JL, Thyssen JP, Schalock PC. Cutaneous and systemic hypersensitivity reactions to metallic implants. Dermatitis 2011; 22: 65-79. 